\title{
Prevalencia de síntomas depresivos, síntomas ansiosos y riesgo de suicidio en estudiantes de medicina de la Pontificia Universidad Católica de Chile.
}

Prevalence of depressive symptoms, anxious symptoms and risk of suicide among medical students of the Pontificia Universidad Católica de Chile

Valentina Kobus $\mathrm{G}^{1}$, María José Calletti $\mathrm{G}^{1}$, Jaime Santander T. ${ }^{2}$

\begin{abstract}
this work is to determine the prevalence of depressive symptoms, anxiety symptoms, and risk of suicide in medical students of the Pontificia Universidad Catolica de Chile, to study their relationship with other psychosocial variables and contrast with previous studies. Methods: a voluntary and self-report survey was designed, which included the Goldberg Abbreviated General Health Questionnaire (GHQ-12), Beck Depression Inventory (BDI), Beck Anxiety Inventory (BAI) and questions taken from the National Health Survey on suicide risk. Results: 559 students answered from a total of 857 (65,2\%). 33,\% observed positive GHQ-12, 66,4\% positive BDI and 34,9\% positive BAI. Regarding suicide risk, 19,1\% have moderate risk and 6,1\% high risk. An association was found with the variables sex, course, fail some branch during the race and stressful life event. Conclusions: the medical students of Pontificia Universidad Católica de Chile have a higher prevalence of depressive symptoms, anxious symptoms, and risk of suicide than the general chilean population. Also, our students have higher rates compared to other studies conducted in the same population during previous years.
\end{abstract}

Key words: Medical students, depression, anxiety,suicide, psychiatry Rev Chil Neuro-Psiquiat 2020; 58 (4):314-323

\section{Introducción}

$\mathrm{L}^{\infty}$ os trastornos neuropsiquiátricos tienen una alta prevalencia en Chile. El $22 \%$ de la población cumple criterios de haber experimentado alguno de ellos en los últimos 12 meses $^{(1)}$ y son la principal fuente de carga de enfermedad en nuestro país ${ }^{(2)}$. Según el últi-

Los autores declaran no tener conflictos de intereses.

Aceptado: 2020/08/06

Recibido: 2020/01/15

1 Interna de Medicina, Escuela de Medicina, Pontificia Universidad Católica de Chile. Santiago, Chile.

2 Departamento de Psiquiatría, Facultad de Medicina, Pontificia Universidad Católica de Chile. Santiago, Chile. 
mo estudio de Carga de Enfermedad y Carga Atribuible realizado, un $23,2 \%$ de los años de vida perdidos por discapacidad o muerte (AVISA) están determinados por condiciones neuropsiquiátricas. La Encuesta Nacional de Salud (ENS) 2016-2017 indica que en la población general existe una prevalencia de $15,8 \%$ de síntomas depresivos y $2,2 \%$ de ideación suicida en los últimos 12 meses $^{(3)}$. Los datos de la Organización Mundial de la Salud (OMS) del año 2016 señalan que en Chile, la tasa de suicidio es 13 por 100.000 habitantes, siendo la segunda causa de muerte de personas entre 15 a 34 años $^{(4)}$. Según el informe del Instituto Médico Legal, de todos los suicidios cometidos durante los años 2011 a 2017 en la Región Metropolitana (RM), un $15,6 \%$ del total corresponde al grupo de 15 a 24 años, cifra que se ha mantenido sin fluctuaciones significativas desde $2011^{(5)}$.

La patología de salud mental no se distribuye del mismo modo en la población. Uno de los grupos de mayor riesgo lo conforman los adolescentes y adultos jóvenes ${ }^{(6)}$. Diversos autores han mostrado preocupación respecto a la salud mental de los estudiantes universitarios, argumentando que el conjunto de actividades académicas puede influir en su bienestar físico y psicológico, llegando incluso a contribuir a la aparición de cuadros ansiosos o depresivos ${ }^{(7)}$. Es así como en esta etapa los individuos pueden experimentar un mayor estrés, pues se encuentran en un período de formación de la identidad, exploración de la sexualidad e intimidad, independencia de los padres y elección de la carrera, todo lo cual, eventualmente, podría repercutir en su salud mental.

Una subpoblación de adultos jóvenes que comúnmente presenta síntomas depresivos, ansiosos y alta tasa de suicidio son los estudiantes de medicina ${ }^{(9)}$. Es así como distintos autores han comparado dichas prevalencias con las presentes en la población general, obteniendo resultados poco alentadores. Rotenstain y cols. ${ }^{(10)}$ realizaron una revisión sistemática publicada en JAMA el año 2017, donde se incluyeron 195 estudios de 43 nacionalidades, con el objetivo de heterogeneizar la prevalencia de síntomas depresivos y riesgo de suicidio en la evidencia existente. En esta revisión se concluye que un $27,2 \%$ de los estudiantes presentaban depresión o síntomas depresivos. A su vez, $11,1 \%$ reportó ideación suicida durante el transcurso de la formación académica. De aquellos estudiantes con screening positivo, solo un $15,7 \%$ se encontraba en tratamiento para su condición. Por otra parte, la revisión sistemática de Dyrbye y cols. concluyen que tanto síntomas depresivos como ansiosos se presentan en mayor proporción que la población general, siendo necesario más estudios sobre las posibles causas y soluciones ${ }^{(11)}$.

En la misma línea, Santander y cols. investigó la prevalencia de síntomas depresivos y ansiosos en estudiantes de medicina de la Pontificia Universidad Católica el año $2007^{(12)}$. Este estudio reportó que $39 \%$ de los estudiantes presentaba riesgo de enfermedad neuropsiquiátrica y un $25 \%$ síntomas depresivos, mayoritariamente de intensidad leve a moderada. Entre las variables asociadas, Santander y cols. establecen que existe mayor prevalencia en mujeres y en aquellos estudiantes que han vivido un evento vital estresante, entendido como un fenómeno económico, social, psicológico y/o familiar de carácter agudo, que produce desadaptación social o distrés psicológico.

El objetivo de este trabajo es determinar la prevalencia de síntomas depresivos, ansiosos y/o riesgo de suicidio en los estudiantes de medicina de la Pontificia Universidad Católica de Chile, con el fin de estudiar su relación con otras variables psicosociales y obtener resultados actuales para contrastar con previos. 


\section{Método}

La presente investigación corresponde a un estudio analítico, individual y transversal. El estudio se llevó a cabo durante la tercera y cuarta semana de Junio de 2019, previa autorización de Dirección de Pregrado de la Escuela de Medicina UC y aprobación por parte del Comité de Ética institucional.

Se invitó a participar a todos los estudiantes de primero a séptimo año de medicina de la Pontificia Universidad Católica de Chile ( $\mathrm{n}=857)$ vía correo electrónico y redes sociales. Previo a la respuesta, los estudiantes aceptaron un consentimiento informado donde se explicaba la finalidad y utilidad del estudio y se aseguraba el anonimato y confidencialidad para el manejo posterior de la información. Respondieron un total de 559 estudiantes, lo que corresponde a un $65,2 \%$ del total. La encuesta se facilitó vía Google Forms, y los datos fueron exportados a Microsoft Excel en primera instancia. El proceso fue supervisado por el equipo investigador.

Para recopilar la información se diseñó una encuesta de autoreporte que incluyó cuatro secciones. La primera sección fue compuesta por 11 preguntas relacionadas con características sociodemográficas. La segunda incluyó las herramientas Cuestionario de Salud General abreviado de Goldberg (GHQ-12) (13) e Inventario de Depresión de Beck (BDI) ${ }^{(14)(15)}$. La tercera corresponde a las preguntas del Inventario de Ansiedad de Beck (BAI). La cuarta estuvo compuesta por preguntas extraídas de la ENS sobre riesgo de suicidio.

El GHQ-12 está diseñado para detectar riesgo de enfermedad ansiosa y/o depresiva, y es aplicable al trabajo clínico, encuestas de población o estudios longitudinales ${ }^{(16)}$. El instrumento consta de 12 preguntas, con 4 alternativas cada una. Dos de las respuestas tienen puntaje igual a 0 , y las dos restantes puntaje igual a 1 . De esta manera, los puntajes mínimo y máximo son 0 y 12 , respectivamente. Se consideran la categoría de sospecha e indicativo como "GHQ-12 positivo", con un puntaje mayor igual a 5 . Se eligió este instrumento porque permite ser aplicado a un grupo importante de personas en un tiempo limitado, porque es de fácil comprensión para el entrevistado y porque está validado en Chile ${ }^{(17)}$. Además, este instrumento ha sido utilizado en dos publicaciones anteriores en estudiantes de medicina de la misma universidad, lo que nos permite comparar resultados ${ }^{(18)}$.

El BDI es un instrumento de 21 preguntas que evalúa la presencia e intensidad de síntomas depresivos. El contenido de los ítems refleja la importancia concedida por los autores al componente cognitivo de la depresión; sólo una tercera parte de las preguntas hace referencia a aspectos fisiológicos o conductuales ${ }^{(12)}$. Ha sido estudiado en población adolescente chilena, evidenciando una alta consistencia interna, aceptable correlación test-retest y discriminación adecuada entre población consultante con sintomatología emocional ansioso-depresiva y población no consultante ${ }^{(19)}$. El rango de la puntuación obtenida es de 0 a 63. La existencia de depresión fue definida como puntaje igual o superior a 10, denominándose "BDI positivo". En cuanto a la intensidad de la depresión, se denominó "leve" un puntaje entre 10 y 18 , "moderada" entre 19 y 29, y "severa" mayor a 30 puntos.

El BAI es una herramienta diseñada para obtener un autorreporte del nivel de ansiedad del participante. Está compuesta por 21 preguntas, que evalúan la presencia e intensidad de síntomas ansiosos. Ha sido estudiada y validada para población universitaria chilena, demostrando un desempeño psicométrico aceptable, con adecuada validez de constructo, convergente y discriminante, así como una sólida consistencia interna ${ }^{(20)}$. El rango de la puntuación obtenida es de 0 a 63. Esta se clasifica en 4 rangos: 0-7 indica 
ansiedad mínima, 8-15 ansiedad leve, 16-25 ansiedad moderada y 26-63 ansiedad grave (Beck y Steer, 1993). Para efectos de este estudio, consideramos como "BAI positivo" los rangos "moderada" y "grave"(21).

En la sección de riesgo de suicidio, se incluyen 3 preguntas extraídas de la ENS 2016-2017: “¿Alguna vez has pensado seriamente en suicidarte?". "En los últimos 12 meses ¿Alguna vez has hecho un plan para suicidarte?" y, "en los últimos 12 meses, ¿Alguna vez has intentado suicidarte?”. Las posibles respuestas fueron: si (1 punto) y no (0 puntos). De tal manera, se sumaron los puntajes y se clasificó a los participantes en bajo riesgo (puntaje total $=0$ ), riesgo moderado (puntaje total $=1$ ) o riesgo alto (puntaje mayor o igual a 2).

Las variables dependientes fueron síntomas depresivos (GHQ-12 positivo o BDI positivo), síntomas ansiosos (BAI positivo) y riesgo de suicidio. Las variables independientes fueron sexo, edad, curso, tipo de financiamiento de educación superior, vivir solo/a, reprobación de algún ramo durante la carrera, diagnóstico previo de patología de salud mental y ocurrencia de eventos vitales estresantes en los últimos 12 meses.

Las variables categóricas se presentan como número de casos y porcentajes. Para comparación de porcentajes se usó test chi-cuadrado. Se consideró significativo todo valor $\mathrm{p}$ menor o igual a 0,05 . Todos los análisis se hicieron usando el programa SPSS 17.

\section{Resultados}

La encuesta fue respondida de manera voluntaria por 559 de un total de 857 estudiantes $(65,2 \%)$. Del total de encuestados, 297 fueron mujeres $(53,1 \%)$ y 262 hombres $(46,9 \%)$. El curso con mayor cantidad de respuestas fue segundo año $(n=99)$ y el con menos sexto año $(n=62)$. El $62,5 \%$ de los estudiantes se encuentran en el rango eta- rio entre 18 a 22 años, un $34,6 \%$ entre 23 a 27 años y un $1,8 \%$ tiene 27 años o más. Un $52,2 \%$ tiene a su familia de origen (padre $y / o$ madre) como fuente única de financiamiento de sus estudios. Por otro lado, el $81,7 \%$ vive con integrantes de su familia nuclear, mientras que un 9,5\% vive solo/a. El 8,1\% ha reprobado algún ramo durante su carrera académica. El 54,6\% de los encuestados declara haber vivido un evento vital estresante en los últimos 12 meses. El 32,9\% ha sido diagnosticado por patología de salud mental y el $24,7 \%$ está actualmente en tratamiento por diagnóstico previo de salud mental.

Del total de estudiantes el 33,6\% obtuvo GHQ-12 positivo, que incluye las categorías de sospecha e indicativa de psicopatología. La variable sexo se asocia en forma significativa con GHQ-12 positivo $(p=0,04)$. Del total de encuestados que obtuvo GHQ-12 positivo ( $\mathrm{n}=188)$, un $61,7 \%(\mathrm{n}=116)$ corresponden a mujeres y un $38,3 \%(n=72)$ a hombres. Al analizar la distribución por cursos, se evidencia asociación significativa con GHQ-12 $(p=0,09)$, siendo segundo año el curso con mayor proporción de estudiantes con GHQ12 positivo $(43,4 \%, n=43)$, seguido por tercer año $(41,9 \%, n=39)$. El curso con menor proporción fue séptimo año $(22,1 \%, \mathrm{n}=17)$. Existe asociación significativa entre tipo de financiamiento de educación superior (crédito, pagado por terceros y mixto) y GHQ-12 positivo $(\mathrm{p}=0.02)$. De todos los estudiantes que se financian vía crédito, ya sea Crédito con Aval del Estado (CAE) u otros $(n=35)$, el 54,3\% $(n=19)$ presentó GHQ-12 positivo. Por otra parte, de todos los estudiantes que son financiados por terceros (ya sea familia directa, extendida o becas) $(\mathrm{n}=410)$, el 32,7\% $(n=134)$ obtuvo GHQ-12 positivo. Existe asociación significativa con haber reprobado algún ramo durante la carrera $(\mathrm{p}=0,024)$. Del total de estudiantes que ha reprobado algún ramo durante la carrera $(\mathrm{n}=45)$, el $48,9 \%(n=22)$ presenta GHQ-12 positivo. Por último, existe asociación significativa 
con haber vivido un evento vital estresante en los últimos 12 meses $(\mathrm{p}<0,001)$. Del total de estudiantes que reportan haber vivido un evento de tales características $(n=305)$, el $74,1 \%(\mathrm{n}=226)$ presentó GHQ-12 positivo. No se encontró asociación significativa con vivir solo/a.

Respecto a síntomas depresivos, un $66,4 \%$ de los estudiantes presentaron BDI positivo. La variable sexo posee asociación significativa, donde un $73,4 \%$ de las mujeres encuestadas presentaron síntomas en contraste con un 58,4\% de los hombres. Al analizar por cursos existe una asociación significativa con valor $p<0.000$. Cabe destacar que el curso con mayor porcentaje de estudiantes con BDI positivo fue segundo año con un $78,6 \%(\mathrm{n}=76)$, seguido por cuarto año con $74,6 \%(n=50)$, sexto año con $72,6 \%(n=45)$ $\mathrm{y}$ tercer año con $72 \%(\mathrm{n}=67)$. Por último, un $74,1 \%$ de los estudiantes con BDI positivo presentaron un evento vital estresante en los últimos 12 meses, existiendo una asociación positiva entre ambas variables con $p<0$. No hubo asociación significativa entre la herramienta y fuente de financiamiento de educación superior, vivir solo/a ni reprobación de algún ramo durante la carrera.

En cuanto a los síntomas ansiosos, un $34,9 \%(\mathrm{n}=195)$ presentó BAI positivo. $\mathrm{La}$ variable sexo posee asociación significativa donde un $25,2 \%$ de los hombres presentó síntomas en comparación a 43,4\% de las mujeres. En cuanto a la asociación por cursos, esta es significativa, donde el $67 \%$ $(n=66)$ de los estudiantes encuestados de segundo año presenta sintomatología ansiosa, seguido de sexto año donde $61,3 \%(n=38)$ presentaron síntomas y luego tercer año con $65,6 \%(n=61)$. Existe asociación significativa entre aquellos estudiantes que viven solo/a y presentar sintomatología ansiosa con $\mathrm{p}<0.049$, donde el $47,2 \%$ de los estudiantes que viven solo/a presentan BAI positivo. Por último, el 44,9\% de los estudiantes que han vivido un evento vital estresante presentan síntomas, existiendo asociación significativa entre ambos. Se descarta asociación entre resultados en BAI y fuente de financiamiento o haber reprobado algún ramo (Tabla 1, Figura 1, Tabla 2).

En relación al riesgo de suicidio, un $19,1 \%$ de los encuestados presenta riesgo moderado y un $6,1 \%$ riesgo alto. Se demostró asociación estadísticamente significativa con valor $\mathrm{p}<0.02$ entre riesgo de suicidio y haber reprobado un ramo durante la carrera. De hecho, de los 45 estudiantes que han reprobado un ramo durante la carrera, un $42,2 \%$ presenta riesgo moderado o alto de suicidio. A su vez, un $32,5 \%$ de los que vivieron un evento vital estresante en los últimos 12 meses, presentan riesgo moderado o alto de suicidio, siendo esta asociación estadísticamente significativa. Se descarta asociación estadísticamente significativa entre riesgo suicida y sexo, curso, fuente de financiamiento de educación superior y vivir solo/a. Cabe destacar que riesgo suicida presenta asociación estadísticamente significativa con valor $\mathrm{p}<0$ con respecto a las 3 herramientas previas. Cabe destacar, que existe asociación estadísticamente significativa entre riesgo leve, moderado o grave de suicidio y las herramientas GHQ-12 positivo, BDI positivo y BAI positivo. (Tabla 3)

Finalmente, un $32,9 \%$ del total de estudiantes encuestados ha sido diagnosticado con alguna patología de salud mental, existiendo una asociación estadísticamente significativa. Un $48,4 \%$ de estudiantes con GHQ-12 positivo, un $82,1 \%$ con BDI positivo y $57,1 \%$ con BAI positivo, contaban con diagnóstico previo. A su vez, $45,7 \%$ de los estudiantes con riesgo de suicidio, presentaba diagnóstico previo.

Del total de estudiantes con diagnóstico, $75 \%$ se encuentra en tratamiento, siendo este en su mayoría psicológico y farmacológico. (Tabla 4) 
Tabla 1. Prevalencia de GHQ-12 positivo, BDI positivo y BAI positivo, por 100 estudiantes, según sexo

\begin{tabular}{|lcccc|}
\hline & Mujeres & Hombres & P & Total \\
\hline GHQ-12 positivo & $116(39,1 \%)$ & $72(27,5 \%)$ & 0.004 & $188(33,6 \%)$ \\
\hline BDI positivo & $218(73,4 \%)$ & $153(58,4 \%)$ & 0.001 & $371(66,4 \%)$ \\
\hline BAI positivo & $129(43,4 \%)$ & $66(25,2 \%)$ & 0.001 & $195(34,9 \%)$ \\
\hline
\end{tabular}

Tabla 2. Prevalencia de GHQ-12 positivo, BDI positivo y BAI positivo, por 100 estudiantes, según ocurrencia de acontecimiento vital importante

\begin{tabular}{|lcccc|}
\hline & $\begin{array}{c}\text { Con acontecimiento } \\
\text { vital }\end{array}$ & $\begin{array}{c}\text { Sin acontecimiento } \\
\text { vital }\end{array}$ & P & Total \\
\hline GHQ-12 positivo & $129(42,3 \%)$ & $59(23,2 \%)$ & 0.001 & $188(33,6 \%)$ \\
\hline BDI positivo & $226(74,1 \%)$ & $145(57,1)$ & 0.001 & $371(66,4 \%)$ \\
\hline BAI positivo & $137(44,9 \%)$ & $58(22,8 \%)$ & 0.001 & $195(34,9 \%)$ \\
\hline
\end{tabular}

Figura 1. Prevalencia de GHQ-12 positivo, BDI positivo y BAI positivo, por 100 estudiantes, según curso.

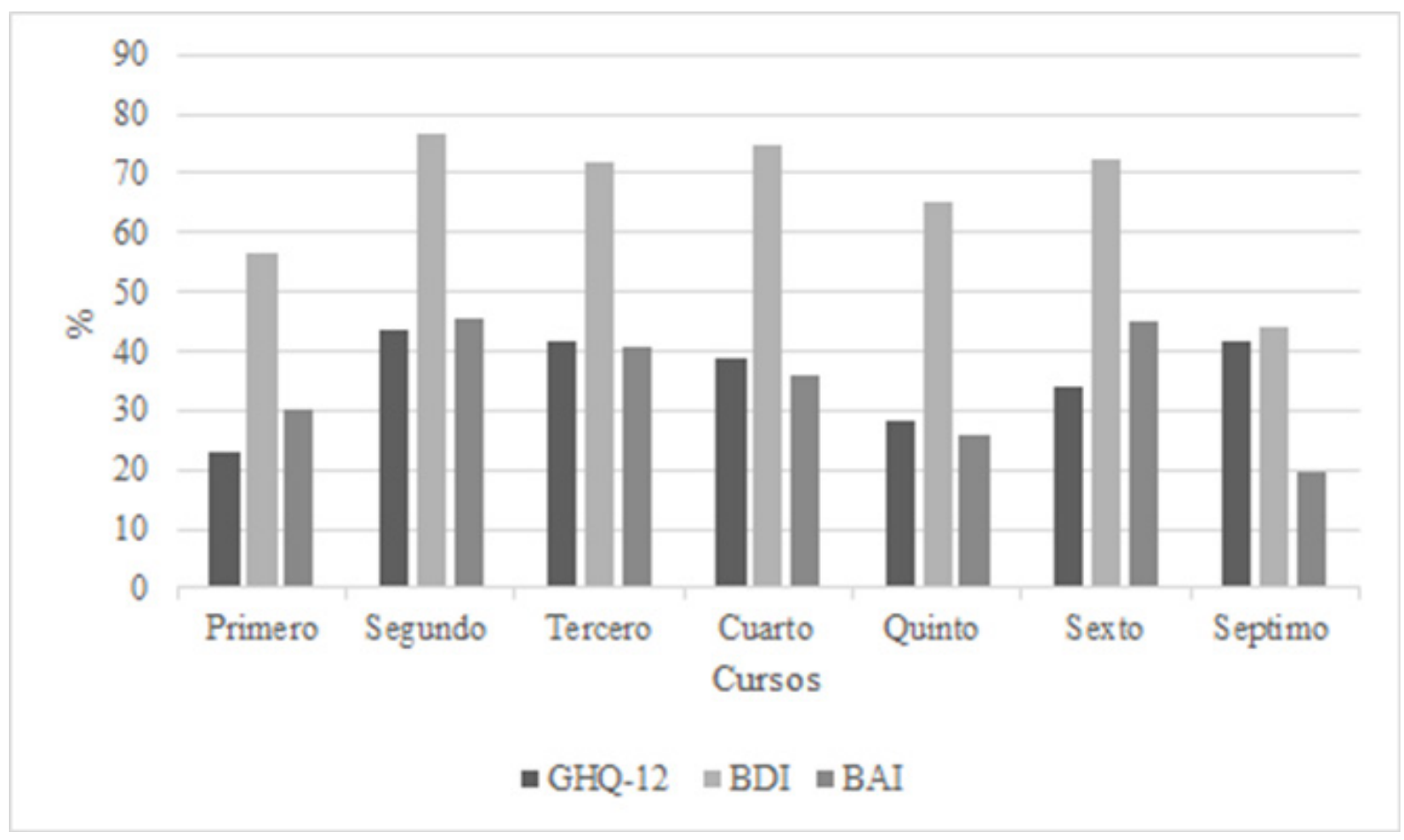


Tabla 3. Prevalencia de riesgo alto, moderado y bajo de suicidio, por 100 estudiantes, según GHQ-12 positivo, BDI positivo y BAI positivo

\begin{tabular}{|lccccc|}
\hline & Riesgo alto & Riesgo moderado & Riesgo bajo & P & Total \\
\hline GHQ-12 positivo & $22(64,7 \%)$ & $53(49,5 \%)$ & $113(27 \%)$ & 0.001 & $188(33,6 \%)$ \\
\hline BDI positivo & $31(91,2 \%)$ & $90(84,1 \%)$ & $250(59,8 \%)$ & 0.001 & $371(66,4 \%)$ \\
\hline BAI positivo & $25(73,5 \%)$ & $62(57,9 \%)$ & $108(25,8 \%)$ & 0.001 & $195(34,9 \%)$ \\
\hline
\end{tabular}

Tabla 4. Prevalencia de GHQ-12 positivo, BDI positivo y BAI positivo, por 100 estudiantes, según presencia o ausencia de tratamiento actual

\begin{tabular}{|lcccc|}
\hline & Si recibe tratamiento & No recibe tratamiento & P & Total \\
\hline GHQ-12 positivo & 65 & 123 & 0.001 & 188 \\
\hline BDI positivo & 117 & 254 & 0.001 & 371 \\
\hline BAI positivo & 78 & 117 & 0.001 & 195 \\
\hline
\end{tabular}

\section{Discusión y conclusiones}

El porcentaje de respuesta en nuestro estudio alcanzó el 65,2\% del total de estudiantes, que es mayor a la cantidad de respuestas que se observan en encuestas sobre el mismo tema y enviadas vía online. No obstante, por el tipo de muestra seleccionado, los datos no pueden ser extrapolados al resto de los estudiantes de otras facultades de la Pontificia Universidad Católica, tampoco a otras universidades ni a la población general.

La tasa de síntomas depresivos, ansiosos y riesgo suicida en la población chilena es menor que la encontrada en el presente estudio, donde se estima que un $15,6 \%$ de la población presenta síntomas depresivos y $2,2 \%$ ideación suicida en los últimos meses, en comparación a nuestros resultados, donde $66,4 \%$ presenta síntomas depresivos y $6,1 \%$ riesgo alto de suicidio. Sin embargo, estos resultados no son estrictamente comparables dado que se utilizaron distintos instrumentos de medición. En cuanto a la literatura internacional, Rotenstain y cols. en su revisión sistemática concluyeron que un $27,2 \%$ de los estudiantes de medicina presentaban depresión o síntomas depresivos, siendo este número menor a lo obtenido en nuestro estudio. En la revisión sistemática, 24 estudios utilizaron BDI como herramienta, donde un $37,5 \%$ de los estudiantes presentaban BDI positivo. En cuanto a riesgo de suicidio, la revisión reporta un riesgo de $11,1 \%$ durante el transcurso de la formación académica, no obstante las herramientas utilizadas en comparación al presente estudio, fueron distintas. Por último, de aquellos estudiantes con screening positivo en la revisión sistemática, sólo un $15,7 \%$ se encontraba en tratamiento para su condición, a diferencia de nuestros resultados donde un $24,7 \%$ del total lo está.

Al comparar los resultados del presente estudio con aquellos de la edición previa publicada por Santander y cols. se aprecian diferencias. Actualmente, un 33,6\% de los estudiantes presentó GHQ-12 positivo, siendo esto menor a lo encontrado en aquella oportunidad, donde un 39\% lo presentó. En contraste, el porcentaje de estudiantes con BDI positivo aumentó de un $25 \%$ a un $66,4 \%$. El riesgo de suicidio no se ha abordado en pu- 
blicaciones anteriores. Por último, en aquella oportunidad un $12 \%$ de los estudiante se encontraban en tratamiento de salud mental, siendo este valor menor a lo encontrado en nuestro estudio. Sobre las variables medidas en tal estudio, persistieron las asociaciones entre síntomas depresivos y ansiosos con ser mujer y haber vivido un evento vital estresante en los últimos 12 meses.

Tanto la literatura internacional como la investigación local han asociado la tasa de depresión en los estudiantes de medicina con características de su personalidad y con la alta demanda académica ${ }^{(22)}$. No existen revisiones sistemáticas que demuestren tal asociación y más bien es planteada como una posible teoría. Sería interesante analizar esta asociación en próximos estudios.

En conclusión, los estudiantes de medicina de la Pontificia Universidad Católica presentan mayor prevalencia de síntomas depresivos, síntomas ansiosos y riesgo de suicidio que la población general chilena. Además, nuestros estudiantes presentan tasas mayores en comparación a otros estudios realizados en la misma población durante años previos. Se propone como próximo desafío estudiar posibles causas asociadas al aumento en cuanto al aumento de síntomas para poder trabajar sobre ellas a futuro.

\section{Resumen}

Introducción: Los trastornos neuropsiquiátricos tienen una alta prevalencia en Chile, siendo los adolescentes y adultos jóvenes uno de los grupos de mayor riesgo. El objetivo de este trabajo es determinar la prevalencia de síntomas depresivos, ansiosos y riesgo de suicidio en los estudiantes de medicina de la Pontificia Universidad Católica de Chile, con el fin de estudiar su relación con otras variables psicosociales y contrastar con estudios previos. Métodos: se diseñó una encuesta voluntaria y de autoreporte, que incluyó el Cuestionario de Salud General abreviado de Goldberg (GHQ-12), Inventario de Depresión de Beck (BDI), Inventario de Ansiedad de Beck (BAI) y preguntas extraídas de la Encuesta Nacional de Salud sobre riesgo de suicidio. Resultados: respondieron 559 estudiantes de un total de 857 (65,2\%). Un 33,6\% presentaron GHQ-12 positivo, 66,4\% BDI positivo y 34,9\% BAI positivo. Sobre riesgo de suicidio, un $19,1 \%$ presenta riesgo moderado y un $6,1 \%$ riesgo alto. Se encontró asociación con las variables sexo, curso, reprobar algún ramo durante la carrera y evento vital estresante. Conclusiones: los estudiantes de medicina de la Pontificia Universidad Católica presentan mayor prevalencia de síntomas depresivos, síntomas ansiosos y riesgo de suicidio que la población general chilena. Además, nuestros estudiantes presentan tasas mayores en comparación a otros estudios realizados en la misma población durante años previos.

Palabras clave: estudiantes de medicina, depresión, ansiedad, suicidio. 


\section{Referencias Bibliográficas}

1.- Vicente B, Kohn R, Rioseco P, Saldivia $\mathrm{S}$, Levav I, Torres $\mathrm{S}$. Lifetime and 12-month prevalence of DSM-III-R disorders in the Chile psychiatric prevalence study. Am J Psychiatry 2006; 163: 1362-1370.

2.- Departamento de Salud Pública, Escuela de Medicina, Pontificia Universidad Católica de Chile. Informe final estudio de carga de enfermedad y carga atribuible. Ministerio de Salud de Chile, Subsecretaría de Salud Pública, Santiago 2008; 1:1-98

3.- Margozzini P, Passi A. Encuesta Nacional de Salud, ENS 2016-2017: un aporte a la planificación sanitaria y políticas públicas en Chile. ARS médica 2018; 43: 30-34..

4.- World Health Organization. World Health Statistics 2016: Monitoring Health for the Sustainable Development Goals. WHO Press 2016; 1:1121.

5.- Nahuelpan E, Varas J. El suicidio en Chile: Análisis del fenómeno desde los datos médico legales. Período 2000-2010. Actualización datos periodo 2011-2017. Unidad de Estadística Servicio Médico Legal, Chile 2018; $1: 1-61$.

6.- Vicente B, Saldivia S, Rioseco P, De La Barra F, Valdivia M, Melipillan $\mathrm{R}$ et al., Epidemiología de trastornos mentales infanto juveniles en la provincia de Cautín. Rev Med de Chile 2010; 138: 965-973.

7.- Feldman L, Goncalves L, Chacón-Puignau G, Zaragoza J, Bagés N, De Pablo J. Relaciones entre estrés académico, apoyo social, salud mental y rendimiento académico en estudiantes universitarios venezolanos. Universitas Psychologica 2008; 7:739-751.
8.- Dávila A, Ruiz R, Moncada L, Gallardo I. Niveles de ansiedad, depresión y percepción de apoyo social en estudiantes de Odontología de la Universidad de Chile. Rev de Psicol 2011; 20:147-172.

9.- $\quad$ Dyrbye LN, West CP, Satele D, Boone S, Tan L, Sloan J et al. Burnout among U.S. medical students, residents, and early career physicians relative to the general U.S. population. Acad Med 2014; 89:51-443.

10.- Rotenstein LS, Ramos M, Torre M, Segal B, Peluso M, Guille C et al. Prevalence of Depression, Depressive Symptoms, and Suicidal Ideation Among Medical Students: : A Systematic Review and Meta-Analysis. JAMA 2016; 316:2214-2236.

11.- Dyrbye L, MD, Thomas M, Shanafelt T. Systematic Review of Depression, Anxiety, and Other Indicators of Psychological Distress Among U.S. and Canadian Medical Students. Acad Med 2006; 81:354-373.

12.- Santander J, Romero MI, Hitschfeld M, Zamora V. Prevalencia de ansiedad y depresión entre los estudiantes de medicina de la Pontificia Universidad Católica de Chile. Rev Chil Neuro-psiquiat 2011; 49:47-55.

13.- Goldberg DP. The detection of psychiatric illness by questionnaire. Maudsley Monographs, 21. London: Oxford University Press, 1972.

14.- Beck AT, Ward CH, Mendelson M, Mock J, Erbaugh J. An inventory for measuring depression. Arch Gen Psychiatry $1961 ; 4: 71-561$.

15.- Beck AT, Rush J, Shaw B, Emery G. Cognitive Therapy of Depression. New York, 1979.

16.- Trucco M, Campusano M, Larraín S. Un cuestionario para detectar desórdenes emocionales. Un estudio de validación preliminar. Rev Chil Neu- 
ro-psiquiatr 1979; 17: 20-5.

17.- Lewis G, Araya R. Is the General Health Questionnaire (12 item) a culturally biased measure of psychiatric disorder? Soc Psychiatry Psychiatr Epidemiol 1995; 30: 5-20.

18.- Benítez C, Quintero J, Torres R. Prevalencia de riesgo de trastornos psiquiátricos en estudiantes de pregrado de la Escuela de Medicina de la P. Universidad Católica de Chile. Rev Med Chile 2001; 19: 8-173.

19.- Melipillán R, Cova F, Rincón P, Valdivia M. Propiedades Psicométricas del Inventario de Depresión de Beck-II en Adolescentes Chilenos. Ter Psicol 2008; 26:59-69.
20.- Antúnez Z, Vinet E. Escalas de Depresión, Ansiedad y Estrés (DASS - 21): Validación de la Versión abreviada en Estudiantes Universitarios Chilenos. Ter Psicol 2012; 30:49-55.

21.- Sanz J, Navarro M. Propiedades psicométricas de una versión española del inventario de ansiedad de beck (BAI) en estudiantes universitarios. Rev Ansiedad y estrés 2003; 9:59-84.

22.- Rotenstein LS, Ramos M, Torre M, Segal B, Peluso M, Guille C et al. Prevalence of Depression, Depressive Symptoms, and Suicidal Ideation Among Medical Students: A Systematic Review and Meta-Analysis. JAMA 2016; 316): 2214-2236.
Correspondencia:

Dr. Jaime Santander T.

+562 22354626 .

jsantand@uc.cl 